\title{
Stability of membrane bound reactions
}

\author{
R. Thul, M. Falcke \\ Hahn-Meitner Institut, Abteilung Theorie, Glienicker Str. 100, D-14109 Berlin, Germany
}

(Dated: October 22, 2004)

\begin{abstract}
We present a novel approach to the dynamics of reactions of diffusing chemical species with species fixed in space e.g. by binding to a membrane. The non-diffusing reaction partners are clustered in areas with a diameter smaller than the diffusion length of the diffusing partner. The activated fraction of the fixed species determines the size of an active sub-region of the cluster. Linear stability analysis reveals that diffusion is one of the major determinants of the stability of the dynamics. We illustrate the model concept with $\mathrm{Ca}^{2+}$ dynamics in living cells, which has release channels as fixed reaction partners. Our results suggest that spatial and temporal structures in intracellular $\mathrm{Ca}^{2+}$ dynamics are caused by fluctuations due to the small number of channels per cluster.
\end{abstract}

PACS numbers: 87.16.Ac, 05.40.-a

Huge chemical gradients occur during dynamic changes of concentrations in living cells. Ions may enter the cytosol through tiny channel pores or chemical species in solution may react with membrane bound partners causing gradients by spatially inhomogeneous production or consumption. An example is the reaction of the membrane bound adenylate cyclase with adenosine trisphosphate (ATP) to form cyclic adenosine monophosphate (cAMP). Usually, reactions are modelled as occurring between species with spatially uniform concentrations neglecting spatial gradients. Here, we show that these gradients have a substantial impact on the dynamics of the reaction. That applies in particular when the membrane bound reaction partners are packed into small clusters with a few tens of individual molecules, which is the case we will consider. The small number of elements per cluster may necessitate stochastic approaches which has been carried out for intracellular $\mathrm{Ca}^{2+}$ dynamics [1-3]. Here, we present an investigation of the deterministic limit of a single cluster.

We consider the cluster to be a membrane patch reacting with the dissolved reaction partner. The small number of molecules per cluster entails a cluster size of the same order of magnitude as the single molecules. Therefore when a membrane bound molecule enters the reaction (e.g. by activation) or leaves it (e.g. by deactivation) the area of the reacting patch changes rather than the concentration of active molecules in a fixed area. Consequently, we model the dynamics of the number of membrane bound molecules taking part in the reaction as a size change of the reacting area. This modeling concept is supported by the results of recent investigations $[4,5]$. Simulations in which the reaction was release from an intracellular store through ion channels showed that reactions saturate at much lower maximal rates when the fraction of reacting membrane bound molecules is described by a changing concentration in a fixed area compared to the rate values obtained with the approach we have chosen [4]. Since cluster diameters are smaller than the diffusion length of the partner in solution, the size of the cluster limits the transport of reaction partners to the reaction and therefore the rate.

We will demonstrate our modeling ideas and results with the example of intracellular $\mathrm{Ca}^{2+}$ dynamics. From the very beginning of the life of an organism to its end, calcium is involved in signaling and control of many processes in almost all of its cells. As a second messenger, it communicates the fertilization across an egg cell and controls apoptosis, it plays an active role in muscle contraction and secretion and has many other functions [6]. $\mathrm{Ca}^{2+}$ fulfills its signaling task by a transient rise of the $\mathrm{Ca}^{2+}$ concentration in the cytosol. That is accomplished by release and uptake of $\mathrm{Ca}^{2+}$ by storage compartments like the endoplasmic reticulum (ER) or mitochondria. These stores are embedded in the cytosol. The flux of $\mathrm{Ca}^{2+}$ between the cytosol and the ER is controlled by inositol-1,4,5-trisphosphate receptor channels $\left(\mathrm{IP}_{3} \mathrm{R}\right)$ for release and the sarco-endoplasmic reticulum $\mathrm{Ca}^{2+}$ ATPase (SERCA) pumps for uptake. Although the receptor channels can be found isolated on the membrane of the ER, they usually build clusters with a diameter of $60-100 \mathrm{~nm}$ comprising between 5 and $40 \mathrm{IP}_{3} \mathrm{Rs}$. Swillens et al. estimated that channels are densely packed inside a cluster, whereas the distance between clusters may range from 3 to $7 \mu \mathrm{m}[5,7]$. Thus cluster distances are two orders of magnitude larger than cluster diameters.

The open probability of $\mathrm{IP}_{3}$ Rs depends on the cytosolic $\mathrm{Ca}^{2+}$ concentration. A moderate increase raises the opening probability whereas a large concentration rise inhibits and closes the release channel. The channel cannot open as long as it is inhibited. Hence, channels are coupled by $\mathrm{Ca}^{2+}$ diffusion in the cytosol since they release what controls their state. The channels within a cluster are strongly coupled since the concentration does not decay on the length scale of a cluster diameter, but the coupling of clusters is only weak.

The small number of channels per cluster and the weak coupling of clusters suggest that fluctuations caused by the random opening and closing of channels are impor- 
tant. That was confirmed by stochastic modeling in the last three years [1-3]. Stochastic models showed spatial and temporal structures even with parameters providing a non-oscillatory or non-excitable deterministic regime. The transition from deterministic to stochastic models was accompanied by a transition from continuous to spatially discrete models. The loss of the oscillatory regime in going from the stochastic to the deterministic approach is explained by the results of this study.

Discrete models require information on the concentration gradients. Simulations of release of $\mathrm{Ca}^{2+}$ through $\mathrm{IP}_{3}$ Rs close to the experimental situation showed that the concentration values at an open channel reach $25-170 \mu \mathrm{M}$, i.e., they are 3-4 orders of magnitude larger than the resting level while the concentration increase at the neighboring cluster reaches only 1.1-2.0 times resting level [4]. Experimental observations like propagating waves and theoretical considerations indicate that the sensitivity of the activating $\mathrm{Ca}^{2+}$ dependence of the opening probability on $\mathrm{Ca}^{2+}$ is tuned to concentration levels close to the resting level and that the inhibitory process has half maximum values of several $\mu \mathrm{M}$. Hence, realistic concentration values at an open channel are likely to saturate inhibition as well as activation by $\mathrm{Ca}^{2+}$. These restrictions on the dynamics have to be taken into account in modeling. Spatially averaged concentration values cannot be used since they change by one order of magnitude only.

Our approach to modeling of intracellular $\mathrm{Ca}^{2+} \mathrm{dy}-$ namics incorporates the extreme localization of release. As mentioned above, channel clusters have a typical diameter below $100 \mathrm{~nm}$. The size of a channel molecule with all its four subunits is about $18 \mathrm{~nm}$ in diameter. When a single channel opens or closes, the fraction of cluster area taking part in conduction of $\mathrm{Ca}^{2+}$ changes to a nonnegligible degree. Open channels are randomly scattered across the cluster area. However, Swillens et al. [5] have shown that it is possible to merge the individual areas of all open $\mathrm{IP}_{3} \mathrm{Rs}$ to a single area of the same size without affecting the concentration dynamics around a cluster. Thus the dynamic change of the number of open channels in a cluster can be described as the dynamic change of the radius $a$ of a concentric conducting area. The value of $a$ is 0 when all channels are closed and equal to the cluster radius $a_{0}$ when all channels are open. Merely for the purpose of simplifying the calculations we consider the cluster to be a sphere rather than a membrane area.

The dynamics of the cytosolic $\mathrm{Ca}^{2+}$ concentration $c$ obeys the partial differential equation:

$$
\dot{c}=D \nabla_{r}^{2} c+k_{l}(E-c)-k_{p} c+k_{c}(E-c) \Theta(a-r) .
$$

Here, $\nabla_{r}^{2}$ denotes the radial part of the Laplace operator in three dimensions. The second term on the r.h.s. of eq. (1) refers to a leak flux, the term $k_{p} c$ describes uptake of $\mathrm{Ca}^{2+}$ by the ER and $k_{c}(E-c)$ release through open channels. $E$ is the $\mathrm{Ca}^{2+}$ concentration in the ER. Release is restricted to the volume $r \leq a$. $\Theta(x)$ is the Heaviside step function.

The radius $a$ of the conducting area is determined by the fraction of channels in the open state. The open state depends on the state of the channel subunits. Each channel consists of four identical subunits. Subunits have binding sites for $\mathrm{Ca}^{2+}$ and $\mathrm{IP}_{3}$. The state of a subunit is determined by the occupation of the binding sites. We use the state scheme of the DeYoung-Keizer (DK) model for the subunit state dynamics. Each subunit has an $\mathrm{IP}_{3}$-binding site, an activating and an inhibiting $\mathrm{Ca}^{2+}$ -binding site. If $\mathrm{IP}_{3}$ is bound to its binding site and a $\mathrm{Ca}^{2+}$ ion to the activating $\mathrm{Ca}^{2+}$-binding site, the subunit is activated. The channel is open, if a minimum number of subunits is activated. Binding of $\mathrm{Ca}^{2+}$ to the inhibiting binding site inhibits the subunit. A subunit can only be activated again upon recovery from inhibition. Eight states of a subunit arise from the three binding sites. We denote the fraction of subunits in a certain state with $p_{i j k}$ where an index is 1 , if a binding sites is occupied, and 0 otherwise. The indices $i, j$ and $k$ represent the $\mathrm{IP}_{3}$ -binding site, activating and inhibiting $\mathrm{Ca}^{2+}$-binding site resp. Therefore the fraction of open subunits is $p_{110}$. We refer to the set $\{p\}$ of the $p_{i j k}$ as gating variables. Their dynamics is of the general form

$$
\dot{p}_{i j k}=g_{i j k}(c(a, t),\{p\}) .
$$

Together with the concentration field $c(r, t)$ the gating variables $\{p\}$ determine the radius $a$ by an algebraic relation. The fraction of open channels is given by the probability that at least three out of four subunits of a channel are activated. The radius $a$ of the volume occupied by this fraction is

$$
a=a_{0} p_{110} \sqrt[3]{4-3 p_{110}} .
$$

Stationary solutions of eqs. (1),(2) are determined by:

$$
\begin{aligned}
& 0=D \nabla_{r}^{2} c+k_{l}(E-c)-k_{p} c+k_{c}(E-c) \Theta(a-r) \\
& 0=g_{i j k}(c(a),\{p\}) .
\end{aligned}
$$

Due to the Heaviside function the equation can be treated separately for $r<a$ and $r>a$. Solving equation (4) for the stationary calcium concentration $c_{s t}$ yields

$$
\begin{aligned}
c_{s t}(r) & =B(a) \frac{\exp \left(k_{2}(r-2 b)\right)-\exp \left(-k_{2} r\right)}{r} \Theta(r-a) \\
& +A(a) \frac{\sinh \left(k_{1} r\right)}{r} \Theta(a-r), \\
& k_{1}^{2}=\frac{k_{l}+k_{p}+k_{c}}{D}, \quad k_{2}^{2}=\frac{k_{l}+k_{p}}{D} .
\end{aligned}
$$

We applied the boundary conditions $c(b)=k_{l} E /\left(k_{l}+\right.$ $k_{p}$ ) at the outer radius $b$ of the cytosol, which corresponds to the base level concentration of the system. The requirement of $c_{s t}(r)$ to be $\mathrm{C}^{1}$ at $r=a$ fixes the constants $A(a)$ and $B(a)$. Using the state scheme of De Young and 


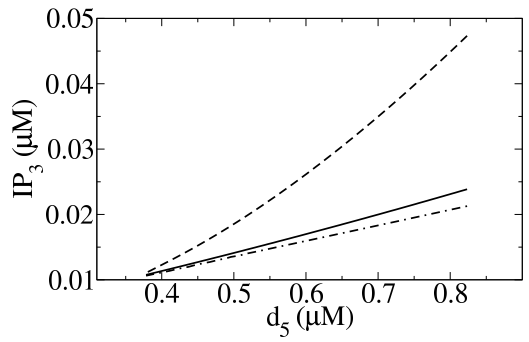

FIG. 1: $\mathrm{IP}_{3}$ concentration of the saddle node bifurcations (solid and chain dotted) and the Hopf bifurcation (dashed) in dependence on $d_{5}$. Parameters read $d_{1}=0.13 \mu M, d_{2}=$ $3 \mu M, d_{3}=0.9434 \mu M, d_{4}=0.4133 \mu M, k_{p}=80 s^{-1}, k_{l}=$ $0.002 s^{-1}, k_{c}=34500 s^{-1}, E=750 \mu M, a_{0}=0.03 \mu m, D=$ $40 \mu m^{2} s^{-1}$

Keizer for a single subunit [? ], the stationary state of $p_{110}$ in dependence on $c$ and the $\mathrm{IP}_{3}$ concentration $I$ is:

$$
p_{110}^{s t}=\frac{d_{2} c_{s t}(a) I}{\left(c_{s t}(a)+d_{5}\right)\left(d_{1} d_{2}+c_{s t}(a) d_{3}+c_{s t}(a) I+d_{2} I\right)} .
$$

Here $d_{1}$ and $d_{3}$ denote the dissociation constants for $\mathrm{IP}_{3}$ when no $\mathrm{Ca}^{2+}$ and when $\mathrm{Ca}^{2+}$ is bound to the inhibiting site, resp. The parameters $d_{2}$ and $d_{4}$ represent the dissociation constants for the inhibiting $\mathrm{Ca}^{2+}$ processes, depending on $\mathrm{IP}_{3}$ binding, and $d_{5}$ is the dissociation constant for the activating $\mathrm{Ca}^{2+}$ site.

The value of the $\mathrm{Ca}^{2+}$ concentration does not vary significantly within the cluster. Hence, we can pick a typical value to enter the dynamics of the gating variables. The value of $c$ at the boundary of the conducting area was chosen (see eq. (7)). The dependence of $p_{110}^{s t}$ on $c(a)$ turns eq. (3) into an implicit function for the value of $a$ at the stationary state: $a=f(a)$. The solution set of this equation determines the stationary states of the system. Saddle node bifurcations can be easily obtained by the condition that the bisection line touches $f(a): 1=f^{\prime}(a)$. Similarly, the stability analysis can be simplified to the solution of an algebraic equation, too. That will be explained in detail in [8]

The DK model assumes that the dynamics of $\mathrm{IP}_{3}$ binding and dissociation is much faster than the dynamics of $\mathrm{Ca}^{2+}$-binding and dissociation. Therefore we eliminate the $\mathrm{IP}_{3}$ dynamics in the following by assuming that it is always in its stationary state. That does not alter the expression for $p_{110}^{s t}$. The original DK model is based on a spatially continuous $\mathrm{IP}_{3} \mathrm{R}$ density and exhibits two Hopf bifurcations bounding an oscillatory regime. We can transform a spatially continuous model into a discrete model by concentrating the flux in an area determined by a typical cluster distance $R$ into an area of typical cluster size $\left(a_{0}\right)$ while conserving the total flux. Consequently, we rescale the original parameter $k_{c}^{D K}$ in [? ] by $k_{c}=k_{c}^{D K} R^{3} / a_{0}^{3}$. With the resulting large value of $k_{c}$, which is close to realistic values of $310^{5} \mu^{-1} s^{-1}$ [4], the oscillatory regime is lost and the model exhibits only a single stationary state which is stable for all values of the $\mathrm{IP}_{3}$ concentration. Lowering $k_{c}$ by several orders of magnitude and thus approaching the original value does not restore the linear stability properties of the continuous model since gradients still occur. That is similar to findings in [9].

However, adaptation of a model designed as spatially continuous to spatially discrete source terms requires more than rescaling of the source strength. The DK model was set up for spatially averaged $\mathrm{Ca}^{2+}$ concentrations which are much lower than concentrations at the channel mouth. That would suggest to adapt dissociation constants for $\mathrm{Ca}^{2+}$-dependent processes. We increased the value of both $\mathrm{Ca}^{2+}$ dissociation constants (dissociation constant $=$ dissociation rate $/$ binding rate constant) for inhibition and activation, $d_{2}$ and $d_{5}$ respectively. Experimental results for different $\mathrm{IP}_{3}$-receptor subtypes provide dissociation constants of the activation process from $77 \mathrm{nM}$ (type 3 receptor [10]) to 309nM (type 1 receptor [11]). We used $d_{5}=0.823 \mu \mathrm{M}$ (see next paragraph). An estimate of the value of the rate constant for the transition from the state with $\mathrm{Ca}^{2+}$ not bound to the activating site to the state with $\mathrm{Ca}^{2+}$ bound to this site, $a_{5}$, can be obtained from puff frequencies as $1(\mu \mathrm{Ms})^{-1}[12]$. The inhibition process showed an effective dissociation constant between $50 \mathrm{nM}$ at low $\mathrm{IP}_{3}$ concentrations and $45 \mu \mathrm{M}$ at high concentrations in experiments by Mak et al. [13]. We adopted the data by Taylor et al. suggesting an effective dissociation constant of approximately $3 \mu \mathrm{M}$ for the inhibition process and effective binding rates, $a_{2}$ and $a_{4}$, for binding of $\mathrm{Ca}^{2+}$ to the inhibitory site of about $0.2(\mu \mathrm{Ms})^{-1}$ [14]. Finally, the diffusion coefficient $D$ needs a brief consideration. Diffusion of free $\mathrm{Ca}^{2+}$ in the cytosol is limited by binding of free $\mathrm{Ca}^{2+}$ to $\mathrm{Ca}^{2+}$-binding proteins especially buffer proteins. Buffering leads to an effective diffusion coefficient in the range of $40 \mu \mathrm{m}^{2} \mathrm{~s}^{-1}$. However, buffers saturate at the values of the concentration of free $\mathrm{Ca}^{2+}$ in the vicinity of an open channel and therefore $\mathrm{Ca}^{2+}$ diffuses in this area with its own diffusion coefficient of $220-300 \mu \mathrm{m}^{2} \mathrm{~s}^{-1}$ . Since buffers are not included in our model we present results for both $D=40 \mu \mathrm{m}^{2} \mathrm{~s}^{-1}$ and $D=220 \mu \mathrm{m}^{2} \mathrm{~s}^{-1}$.

Dynamic regimes of the model in dependence on the $\mathrm{IP}_{3}$ concentration and $d_{5}$ are shown in Fig. 1. There are two saddle node bifurcation lines terminating in a cusp. A Hopf bifurcation occurs above the two saddle node bifurcations. Oscillations can be found at $\mathrm{IP}_{3}$ concentrations bounded by the Hopf bifurcation and a bifurcation occurring between the Hopf bifurcation and the lower saddle node bifurcation. The type of this bifurcation is still under investigation but is probably homoclinic. Fig. 1 demonstrates that oscillations do not occur at values of $d_{5}$ suggested by measurements, since the activation process completely saturates at the concentration values occurring at an open channel. Hence, changing dissociation constants of the original DK model to larger experimentally supported values did not restore oscillations. However, even if the cluster dynamics oscil- 
lated, these oscillations would not be the experimentally observed ones. To demonstrate that we need to choose parameter values allowing for oscillations. Therefore we use a value of $k_{c}$ large enough to provide realistic concentration values at the releasing cluster (i.e. larger than $25 \mu \mathrm{M}[4])$, if a large fraction of channels is open, but small enough to still see a variety of dynamic regimes, and a value of $d_{5}$ allowing for oscillations (see caption Fig. 1). Stationary states with these parameter values are presented in Fig 2. There is just a single stationary state for almost all $\mathrm{IP}_{3}$ concentrations. An oscillatory regime exists close to the bistable area (see Fig. 2). We did not find limit cycles where the stationary state is stable. Hence, the discrete DK model does not have an oscillatory regime of experimentally relevant extension. Besides the size of the oscillatory regime, there
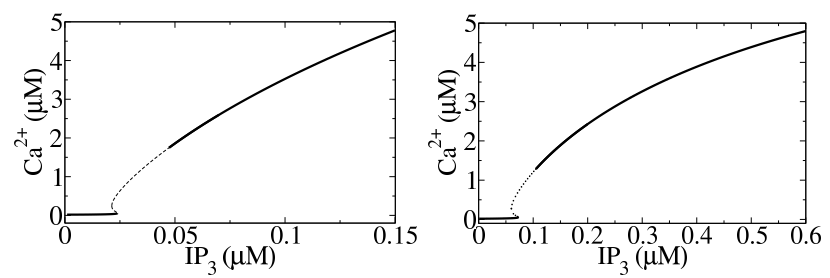

FIG. 2: Stationary values of the $\mathrm{Ca}^{2+}$ concentration for $D=$ $40 \mu \mathrm{m}^{2} \mathrm{~s}^{-1}$ (left) and $D=220 \mu \mathrm{m}^{2} \mathrm{~s}^{-1}$ (right). Solid lines denote linearly stable fixed points, dotted linearly unstable points. Parameters as in Fig 1 and $d_{5}=0.8234 \mu M, a_{2}=$ $a_{4}=0.2(\mu M s)^{-1}, a_{5}=1(\mu M s)^{-1}$.
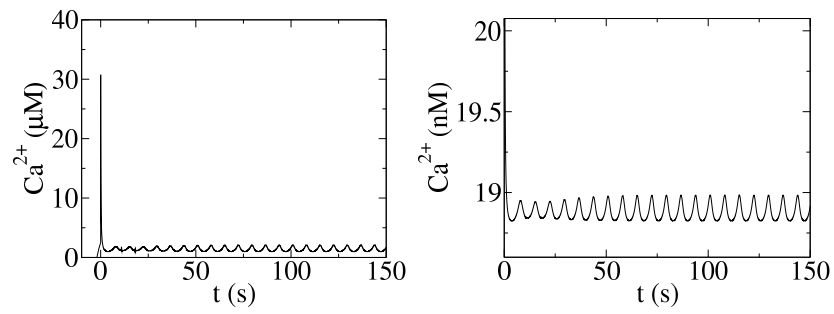

FIG. 3: Oscillation of the $\mathrm{Ca}^{2+}$ concentration at $r=0 \mu m$ (left) and $r=1.588 \mu m$ (right). Note the difference in the order of magnitude for the amplitude and mean. Parameters as in Fig 2 and $D=40 \mu m^{2} s^{-1}$.

is another observation suggesting that these oscillations are not the experimentally observed global oscillations in cells. Fig. 3 shows oscillations of the $\mathrm{Ca}^{2+}$ concentration. The initial transient illustrates that realistic concentration values at the cluster are reached for a large fraction of open channels. The amplitude of the oscillations at the releasing cluster is much smaller than the initial peak. It is even more damped down to less than $1 \mathrm{nM}$ in a distance of $1.6 \mu \mathrm{m}$ from the cluster. That bulk amplitude is too small to represent the observed global oscillations. The oscillation amplitude at the releasing cluster is in the order of magnitude of the dissociation constant of the inhibitory process as is to be expected for such a sinusoidal oscillation. That amplitude in the range of dissociation constants - and the small bulk amplitudes - will apply to other models than the DK model, too. Hence, not only is the oscillatory regime too small in parameter space to be the experimentally observed regime but also is it found at unphysiological values. Moreover the bulk amplitudes are too small.

In summary, the above results strongly suggest that discrete deterministic models of intracellular $\mathrm{Ca}^{2+}$ dynamics including no other control of the channel state but activation by $\mathrm{IP}_{3}$, activation and inhibition by $\mathrm{Ca}^{2+}$ do not show the experimentally observed oscillatory regime as already suggested in [2]. Vice versa, comparison of the bifurcation scheme of the deterministic Li-Rinzel model with simulations of its stochastic counterpart demonstrated that the deterministic regime cannot be concluded from the stochastic behavior [15]. Oscillation-like behavior is reintroduced in intracellular $\mathrm{Ca}^{2+}$ dynamics by the fluctuations resulting from the randomness of binding and dissociation of $\mathrm{Ca}^{2+}$ and $\mathrm{IP}_{3}$ at the regulatory binding sites [1-3]. This is also illustrated by array enhanced coherence resonance where stochasticity can induce global oscillations in non-oscillatory systems [16]. Hence, fluctuations drive the spatio-temporal structure formation and render intracellular $\mathrm{Ca}^{2+}$ dynamics a truly stochastic medium.

[1] M. Falcke, L. Tsimring, and H. Levine, Phys.Rev.E 62, 2636 (2000).

[2] M. Falcke, Biophys.J. 84, 42 (2003).

[3] M. Bär, M. Falcke, L. Tsimring, and H. Levine, Phys.Rev.Lett. 84, 5664 (2000).

[4] R. Thul and M. Falcke, Biophys.J. 86, 2660 (2004).

[5] S. Swillens, G. Dupont, and P. Champeil, Proc.Nat.Acad.Sci.USA 96, 13750 (1999).

[6] M. Berridge, P. Lipp, and M. Bootmann, Nature Rev. Mol. Cell Biol. 1, 11 (2000).

[7] J. Marchant and I. Parker, The EMBO Journal 20, 65 (2001).

[8] R. Thul and M. Falcke, Biophysical Journal 86, 2660 (2004).

[9] J. Sneyd and J. Sherrat, SIAM J.Appl.Math. 57, 73 (1997).

[10] D. Mak, S. McBride, and J. Foskett, J.Gen.Physiol. 117, 435 (2001).

[11] J. Ramos-Franco, S. Caenepeel, M. Fill, and G. Mignery, Biophys.J. 75, 2783 (1998).

[12] Y. Yao and I. Parker, J.Physiol.(Cambridge) 482, 533 (1995).

[13] D. Mak, S. McBride, and J. Foskett, J.Gen.Physiol. 122, 583 (2003).

[14] C. Adkins and C. Taylor, Current Biology 9, 1115 (1999).

[15] P. Jung and J. Shuai, Europhys.Lett. 56, 29 (2001).

[16] S. Coombes and Y. Timofeeva, Phys.Rev.E 68, 021915 (2003). 